\title{
An Investigation of an Integral Equation Involving Convex-Concave Nonlinearities
}

\author{
Ravi P. Agarwal 1,+(D), Mohamed Jleli ${ }^{2,+}$ (D) and Bessem Samet ${ }^{2, *,+(\mathbb{D})}$ \\ 1 Department of Mathematics, Texas A \& M University-Kingsville, Kingsville, TX 78363, USA; \\ Ravi.Agarwal@tamuk.edu \\ 2 Department of Mathematics, College of Science, King Saud University, P.O. Box 2455, \\ Riyadh 11451, Saudi Arabia; jleli@ksu.edu.sa \\ * Correspondence: bsamet@ksu.edu.sa \\ + These authors contributed equally to this work.
}

check for updates

Citation: Agarwal, R.P.; Jleli, M.; Samet, B. An Investigation of an Integral Equation Involving Convex-Concave Nonlinearities. Mathematics 2021, 9, 2372. https:// doi.org/10.3390/math9192372

Academic Editors: Denis N. Sidorov and Massimiliano Ferrara

Received: 23 June 2021

Accepted: 13 September 2021

Published: 24 September 2021

Publisher's Note: MDPI stays neutral with regard to jurisdictional claims in published maps and institutional affiliations.

Copyright: (c) 2021 by the authors. Licensee MDPI, Basel, Switzerland. This article is an open access article distributed under the terms and conditions of the Creative Commons Attribution (CC BY) license (https:/ / creativecommons.org/licenses/by/ $4.0 /)$.
Abstract: We investigate the existence and uniqueness of positive solutions to an integral equation involving convex or concave nonlinearities. A numerical algorithm based on Picard iterations is provided to obtain an approximation of the unique solution. The main tools used in this work are based on partial-ordering methods and fixed-point theory. Our results are supported by examples.

Keywords: integral equation; convex-concave nonlinearities; positive solution

\section{Introduction}

Our aim in this paper is to study the nonlinear integral equation

$$
\vartheta(\sigma)=\xi(\sigma, \vartheta(\sigma))+\int_{\sigma-\ell}^{\sigma} \mu(\sigma, s) \lambda(s, \vartheta(s)) d s, \quad \sigma \in \mathbb{R},
$$

where $\ell>0$ is a constant. When $\xi \equiv 0$ and $\mu \equiv 1,(1)$ reduces to

$$
\vartheta(\sigma)=\int_{\sigma-\ell}^{\sigma} \lambda(s, \vartheta(s)) d s, \quad \sigma \in \mathbb{R}
$$

Equation (2) was proposed in [1] as a mathematical model to analyze the observed periodic outbreaks of certain infectious diseases. Namely, for a given population, $\vartheta(\sigma), \ell$, and $\lambda(\sigma, \vartheta(\sigma))$ represent, respectively, the proportion of infectious individuals at time $\sigma$, the length of time for which an individual is infective, and the proportion of new infective individuals per unit of time.

Several investigations of Equation (2) have been carried out. In [1], sufficient conditions ensuring the existence of nontrivial periodic solutions to (2), as well as sufficient conditions for which all solutions to (2) approach zero as $\sigma \rightarrow \infty$, were provided. In [2,3], using Krasnosel'skii-type fixed point theorems, the existence of at least one nontrivial periodic solution to (2) was proved under certain conditions on $\lambda$. The same question was investigated in [4] using fixed-point index theory. In [5,6], the question of points of bifurcations of positive periodic solutions to (2) was studied. For other contributions related to the study of (2), see, e.g., [7-10] and the references therein.

Various interesting contributions dealing with generalized variants of (2) have been performed by many authors. In [11], the existence of positive almost periodic solutions to integral equations of the form

$$
\vartheta(\sigma)=\int_{\sigma-\ell(\vartheta(\sigma))}^{\sigma} \lambda(s, \vartheta(s)) d s, \quad \sigma \in \mathbb{R}
$$


has been studied. In [12], the neutral integral equation

$$
\vartheta(\sigma)=\gamma \vartheta(\sigma-\ell)+(1-\gamma) \int_{\sigma-\ell}^{\sigma} \lambda(s, \vartheta(s)) d s, \quad \sigma \in \mathbb{R},
$$

has been considered. In [13], the existence of multiple periodic solutions to integral equations of the form

$$
\vartheta(\sigma)=\int_{\sigma-\ell}^{\sigma} \mu(\sigma, s) \lambda(s, \vartheta(s)) d s, \quad \sigma \in \mathbb{R},
$$

has been investigated using various fixed-point theorems. For more contributions related to generalized variants of (2), see, e.g., [14-22] and the references therein.

In [23], sufficient conditions for the existence of a principal solution to a nonlinear Volterra integral equation of the second kind on the half-line and on a finite interval have been derived. Furthermore, a method for computing the boundary of an interval outside of which the solution can blow up has been proposed (see also [24]). In [25], the local solvability and blow-up of solutions to an abstract nonlinear Volterra integral equation have been investigated. Recently, in [26], the authors proposed a new method and a tool to validate the numerical results of Volterra integral equations with discontinuous kernels in linear and nonlinear forms obtained from the Adomian decomposition method.

In this paper, Equation (1) is investigated. Namely, using partial-ordering methods and a fixed-point theorem for monotone and convex/concave operators defined in a normal solid cone, we derive sufficient conditions, ensuring the existence and uniqueness of positive solutions. Moreover, in order to approximate the solution, a numerical algorithm based on Picard iterations is provided.

The main tools of partial-ordering methods and fixed-point theory that will be used in this paper are presented in Section 2. The main results, as well as their proofs, are presented in Section 3. Finally, some examples are studied in Section 4.

\section{Preliminaries}

Let $\mathbb{B}$ be a Banach space over $\mathbb{R}$ with respect to a certain norm $\|\cdot\|_{\mathbb{B}}$. We denote, by $0_{\mathbb{B}}$, the zero vector of $\mathbb{B}$. Let $\mathcal{C} \subset \mathbb{B}\left(\mathcal{C} \neq\left\{0_{\mathbb{B}}\right\}\right)$ be nonempty, closed, and convex. We say that $\mathcal{C}$ is a cone in $\mathbb{B}$, if

- $\quad \alpha \mathcal{C} \subset \mathcal{C}$ for all $\alpha \geq 0$;

- $\quad-\mathcal{C} \cap \mathcal{C}=\left\{0_{\mathbb{B}}\right\}$.

Here, for $\alpha \in \mathbb{R}, \alpha \mathcal{C}$ denotes the subset of $\mathbb{B}$ defined by

$$
\alpha \mathcal{C}=\{\alpha z: z \in \mathcal{C}\}
$$

Let $\mathcal{C}$ be a cone in $\mathbb{B}$. Then $\mathcal{C}$ induces a partial-order $\preceq_{\mathcal{C}}$ in $\mathbb{B}$ defined by

$$
x \preceq_{\mathcal{C}} y \Longleftrightarrow y-x \in \mathcal{C},
$$

for all $x, y \in \mathbb{B}$. We use the notation $x \prec_{\mathcal{C}} y$ to indicate that $x \preceq_{\mathcal{C}} y$ and $x \neq y$. For $x \prec_{\mathcal{C}} y$, the segment $[x, y]$ is defined by

$$
[x, y]=\left\{z \in \mathbb{B}: x \preceq_{\mathcal{C}} z \preceq_{\mathcal{C}} y\right\} .
$$

The notation $x \preceq_{\mathcal{C}} y$ indicates that $y-x \in \mathcal{C}$, where $\mathcal{C}$ is the interior of $\mathcal{C}$. If $\mathcal{C} \neq \varnothing$, We say that $\mathcal{C}$ is a solid cone. We say that $\mathcal{C}$ is normal, if there exists $\rho \geq 1$ such that

$$
0_{\mathbb{B}} \preceq_{\mathcal{C}} x \preceq_{\mathcal{C}} y \Longrightarrow\|x\|_{\mathbb{B}} \leq \rho\|y\|_{\mathbb{B}},
$$

for all $x, y \in \mathbb{B}$.

Let $S: A \subset \mathbb{B} \rightarrow \mathbb{B}$ be a given operator. Then, 
(i) $S$ is nondecreasing, if

$$
x, y \in A, x \preceq_{\mathcal{C}} y \Longrightarrow S x \preceq_{\mathcal{C}} S y
$$

(ii) $S$ is nonincreasing, if

$$
x, y \in A, x \preceq_{\mathcal{C}} y \Longrightarrow S x \succeq_{\mathcal{C}} S y .
$$

(iii) $S$ is convex, if $A$ is a convex set and

$$
\eta \in(0,1), x, y \in A \Longrightarrow S(\eta x+(1-\eta) y) \preceq_{\mathcal{C}} \eta S x+(1-\eta) S y .
$$

(iv) $S$ is concave, if $A$ is a convex set and

$$
\eta \in(0,1), x, y \in A \Longrightarrow S(\eta x+(1-\eta) y) \preceq_{\mathcal{C}} \eta S x+(1-\eta) S y .
$$

Lemma 1 (see [27]). Suppose that $\mathcal{C}$ is a normal solid cone and $S:[\bar{x}, \bar{y}] \rightarrow \mathbb{B}$ is increasing, where $\bar{x}, \bar{y} \in \mathbb{B}$ and $\bar{x} \prec_{\mathcal{C}} \bar{y}$. Assume that one of the following conditions is satisfied:

(i) $S$ is concave, $S \bar{x} \succeq_{\mathcal{C}} \bar{x}$ and $S \bar{y} \preceq_{\mathcal{C}} \bar{y}$.

(ii) $S$ is convex, $S \bar{x} \succeq_{\mathcal{C}} \bar{x}$ and $S \bar{y} \preceq_{\mathcal{C}} \bar{y}$.

Then,

(I) S has a unique fixed point $z \in[\bar{x}, \bar{y}]$.

(II) There exist $\gamma>0$ and $0<\theta<1$, such that for all $z_{0} \in[\bar{x}, \bar{y}]$, the sequence $\left\{z_{n}\right\}_{n \geq 0}$ defined by

$$
z_{n+1}=S z_{n}, \text { for all } n
$$

converges to $z$ and satisfies

$$
\left\|z_{n}-z\right\|_{\mathbb{B}} \leq \gamma \theta^{n}, \text { for all } n
$$

\section{Existence and Uniqueness Results}

3.1. Case 1. $\xi(\sigma, \cdot)$ and $\lambda(\sigma, \cdot)$ Are Concave

Theorem 1. Assume that the following conditions hold:

(i) $\mu \in C(\mathbb{R} \times \mathbb{R},[0, \infty))$.

(ii) There exist $0 \leq m_{\ell}<M_{\ell}$ such that

$$
m_{\ell} \leq \int_{\sigma-\ell}^{\sigma} \mu(\sigma, s) d s \leq M_{\ell}, \quad \sigma \in \mathbb{R}
$$

(iii) There exist $0<h<H$ such that $\xi, \lambda \in C(\mathbb{R} \times[h, H], \mathbb{R})$.

(iv) For all $\sigma \in \mathbb{R}$, the functions $\xi(\sigma, \cdot), \lambda(\sigma, \cdot):[h, H] \rightarrow \mathbb{R}$ are concave and nondecreasing.

(v) There exist $\alpha_{\xi}, \beta_{\xi} \in \mathbb{R}$ and $\alpha_{\lambda}, \beta_{\lambda} \geq 0$, such that

$$
\xi(\sigma, h) \geq \alpha_{\xi} h, \quad \xi(\sigma, H) \leq \beta_{\xi} H, \quad \lambda(\sigma, h) \geq \alpha_{\lambda} h, \quad \lambda(\sigma, H) \leq \beta_{\lambda} H,
$$

for all $\sigma \in \mathbb{R}$.

(vi) $\alpha_{\xi}+\alpha_{\lambda} m_{\ell}>1$ and $\beta_{\xi}+\beta_{\lambda} M_{\ell} \leq 1$.

Then, the integral Equation (1) has a unique continuous solution $\vartheta^{*}$, such that

$$
h \leq \vartheta^{*}(\sigma) \leq H, \quad \sigma \in \mathbb{R}
$$

Moreover, there exist $\gamma>0$ and $0<\theta<1$, such that for any continuous function $z_{0}$ satisfying $h \leq z_{0}(\sigma) \leq H, \sigma \in \mathbb{R}$, the sequence $\left\{z_{n}\right\}_{n \geq 0}$ defined by

$$
z_{n+1}(\sigma)=\xi\left(\sigma, z_{n}(\sigma)\right)+\int_{\sigma-\ell}^{\sigma} \mu(\sigma, s) \lambda\left(s, z_{n}(s)\right) d s, \quad \sigma \in \mathbb{R}
$$


converges uniformly to $\vartheta^{*}$ and satisfies

$$
\sup _{\sigma \in \mathbb{R}}\left|z_{n}(\sigma)-\vartheta^{*}(\sigma)\right| \leq \gamma \theta^{n}, \text { for all } n
$$

Proof. Let us introduce the set

$$
\mathbb{B}=\left\{\vartheta \in C(\mathbb{R}): \sup _{\sigma \in \mathbb{R}}|\vartheta(\sigma)|<\infty\right\} .
$$

Then $\left(\mathbb{B},\|\cdot\|_{\mathcal{B}}\right)$ is a Banach space, where

$$
\|\vartheta\|_{\mathbb{B}}=\sup _{\sigma \in \mathbb{R}}|\vartheta(\sigma)|, \quad \vartheta \in \mathbb{B}
$$

Let

$$
\mathcal{C}=\{\vartheta \in \mathbb{B}: \vartheta(\sigma) \geq 0, \sigma \in \mathbb{R}\} .
$$

Then, $\mathcal{C}$ is a normal solid cone in $\mathbb{B}$, and its interior is given by

$$
\stackrel{\circ}{C}=\left\{\vartheta \in \mathbb{B}: \inf _{\sigma \in \mathbb{R}} \vartheta(\sigma)>0\right\} .
$$

The partial order induced by $\mathcal{C}$ is defined by

$$
x \preceq_{\mathcal{C}} y \Longleftrightarrow x(\sigma) \leq y(\sigma), \sigma \in \mathbb{R},
$$

for all $x, y \in \mathbb{B}$. Let $\bar{x} \equiv h$ and $\bar{y} \equiv H$. For $\vartheta \in[\bar{x}, \bar{y}]$, let

$$
S(\vartheta)(\sigma)=\xi(\sigma, \vartheta(\sigma))+\int_{\sigma-\ell}^{\sigma} \mu(\sigma, s) \lambda(s, \vartheta(s)) d s, \quad \sigma \in \mathbb{R} .
$$

We shall prove that

$$
S([\bar{x}, \bar{y}]) \subset \mathbb{B} .
$$

Let $\vartheta \in[\bar{x}, \bar{y}]$. We first show that $S \vartheta \in C(\mathbb{R})$. Let $\sigma_{0} \in \mathbb{R}$ and $\sigma_{0}-\delta<\sigma<\sigma_{0}+\delta$ for some $\delta>0$. Then,

$$
\int_{\sigma-\ell}^{\sigma} \mu(\sigma, s) \lambda(s, \vartheta(s)) d s=\int_{\sigma_{0}-\delta-\ell}^{\sigma_{0}+\delta} \mathbb{X}_{[\sigma-\ell, \sigma]}(s) \mu(\sigma, s) \lambda(s, \vartheta(s)) d s,
$$

where $\mathbb{X}_{[\sigma-\ell, \sigma]}$ is the characteristic function of $[\sigma-\ell, \sigma]$. By (i), we have

$\lim _{\sigma \rightarrow \sigma_{0}} \mathbb{X}_{[\sigma-\ell, \sigma]}(s) \mu(\sigma, s) \lambda(s, \vartheta(s))=\mathbb{X}_{\left[\sigma_{0}-\ell, \sigma_{0}\right]}(s) \mu\left(\sigma_{0}, s\right) \lambda(s, \vartheta(s)), \quad \sigma_{0}-\delta-\ell \leq s \leq \sigma_{0}+\delta$

and

$$
\left|\mathbb{X}_{[\sigma-\ell, \sigma]}(s) \mu(\sigma, s) \lambda(s, \vartheta(s))\right| \leq C_{k} \mid \lambda\left(s, \vartheta(s) \mid, \quad \sigma_{0}-\delta-\ell \leq s \leq \sigma_{0}+\delta,\right.
$$

where

$$
C_{k}=\sup \left\{\mu\left(\sigma_{1}, \sigma_{2}\right):\left(\sigma_{1}, \sigma_{2}\right) \in\left[\sigma_{0}-\delta, \sigma_{0}+\delta\right] \times\left[\sigma_{0}-\delta-\ell, \sigma_{0}+\delta\right]\right\} .
$$

Moreover, by (iii) we have

$$
\int_{\sigma_{0}-\delta-\ell}^{\sigma_{0}+\delta}|\lambda(s, \vartheta(s))| d s<\infty
$$


Then by the dominated convergence theorem, it holds that

$$
\begin{aligned}
\lim _{\sigma \rightarrow \sigma_{0}} \int_{\sigma-\ell}^{\sigma} \mu(\sigma, s) \lambda(s, \vartheta(s)) d s & =\int_{\sigma_{0}-\delta-\ell}^{\sigma_{0}+\delta} \mathbb{X}_{\left[\sigma_{0}-\ell, \sigma_{0}\right]}(s) \mu(\sigma, s) \lambda(s, \vartheta(s)) d s \\
& =\int_{\sigma_{0}-\delta}^{\sigma_{0}} \mu\left(\sigma_{0}, s\right) \lambda(s, \vartheta(s)) d s
\end{aligned}
$$

which shows the continuity of the function $\sigma \mapsto \int_{\sigma-\ell}^{\sigma} \mu(\sigma, s) \lambda(s, \vartheta(s)) d s$ at $\sigma_{0}$. Since $\sigma_{0}$ is arbitrary, the continuity holds in $\mathbb{R}$. On the other hand, it follows from the continuity of $\xi$ (see (iii)) that the function $\sigma \mapsto \xi(\sigma, \vartheta(\sigma))$ is continuous in $\mathbb{R}$. Then $S \vartheta \in C(\mathbb{R})$. Next, we show that $S \vartheta$ is a bounded function. Using that $\mu \geq 0$, the monotone properties of $\xi$ and $\lambda$ (see (iv)), (ii) and (v), we obtain

$$
\alpha_{\xi} \bar{x} \leq \xi(\sigma, \bar{x}) \leq \xi(\sigma, \vartheta(\sigma)) \leq \xi(\sigma, \bar{y}) \leq \beta_{\xi} \bar{y}, \quad \sigma \in \mathbb{R}
$$

and

$$
\alpha_{\lambda} \bar{x} m_{\ell} \leq \int_{\sigma-\ell}^{\sigma} \mu(\sigma, s) \lambda(s, \vartheta(s)) d s \leq \beta_{\lambda} \bar{y} M_{\ell}, \quad \sigma \in \mathbb{R} .
$$

Combining (4) with (5), we obtain

$$
\bar{x}\left(\alpha_{\xi}+\alpha_{\lambda} m_{\ell}\right) \leq(S \vartheta)(\sigma) \leq \bar{y}\left(\beta_{\xi}+\beta_{\lambda} M_{\ell}\right), \quad \sigma \in \mathbb{R},
$$

which shows that $S \vartheta$ is bounded. Therefore, $S \vartheta \in \mathbb{B}$ and (3) is proved.

Next, we show that $S:[\bar{x}, \bar{y}] \rightarrow \mathbb{B}$ is nondecreasing with respect to $\preceq_{\mathcal{C}}$. Let $x, y \in[\bar{x}, \bar{y}]$ be such that $x(\sigma) \leq y(\sigma)$ for all $\sigma \in \mathbb{R}$. Using the fact that $\mu \geq 0$ and the monotone properties of $\xi$ and $\lambda$, it holds that

$$
\xi(\sigma, x(\sigma)) \leq \xi(\sigma, y(\sigma)), \quad \sigma \in \mathbb{R}
$$

and

$$
\int_{\sigma-\ell}^{\sigma} \mu(\sigma, s) \lambda(s, x(s)) d s \leq \int_{\sigma-\ell}^{\sigma} \mu(\sigma, s) \lambda(s, y(s)) d s, \quad \sigma \in \mathbb{R} .
$$

Then, by (6) and (7), we get

$$
\xi(\sigma, x(\sigma))+\int_{\sigma-\ell}^{\sigma} \mu(\sigma, s) \lambda(s, x(s)) d s \leq \xi(\sigma, y(\sigma))+\int_{\sigma-\ell}^{\sigma} \mu(\sigma, s) \lambda(s, y(s)) d s, \quad \sigma \in \mathbb{R},
$$

i.e., $(S x)(\sigma) \leq S(y)(\sigma)$ for all $\sigma \in \mathbb{R}$. Consequently, the operator $S:[\bar{x}, \bar{y}] \rightarrow \mathbb{B}$ is nondecreasing.

Next, we show the concavity of the operator $S$. Let $0<\eta<1$ and $x, y \in[\bar{x}, \bar{y}]$. By the concavity of $\xi(\sigma, \cdot)$ and $\lambda(\sigma, \cdot), \sigma \in \mathbb{R}$ (see (iv)) and, using the fact that $\mu \geq 0$, we obtain

$$
\xi(\sigma, \eta x(\sigma)+(1-\eta) y(\sigma)) \geq \eta \xi(\sigma, x(\sigma))+(1-\eta) \xi(\sigma, y(\sigma))
$$

and

$$
\begin{aligned}
\int_{\sigma-\ell}^{\sigma} \mu(\sigma, s) \lambda(s, \eta x(s)+(1-\eta) y(s)) d s \geq & \eta \int_{\sigma-\ell}^{\sigma} \mu(\sigma, s) \lambda(s, x(s)) d s \\
& +(1-\eta) \int_{\sigma-\ell}^{\sigma} \mu(\sigma, s) \lambda(s, y(s)) d s,
\end{aligned}
$$

for all $\sigma \in \mathbb{R}$. Hence, it follows from (8) and (9) that

$$
S(\eta x+(1-\eta) y)(\sigma) \geq \eta(S x)(\sigma)+(1-\eta)(S y)(\sigma), \quad \sigma \in \mathbb{R},
$$

which proves that $S:[\bar{x}, \bar{y}] \rightarrow \mathbb{B}$ is concave. 
Now, using that $\mu \geq 0$, (ii), (v) and (vi), for all $\sigma \in \mathbb{R}$, we obtain

$$
\begin{aligned}
(S \bar{x})(\sigma) & =\xi(\sigma, h)+\int_{\sigma-\ell}^{\sigma} \mu(\sigma, s) \lambda(s, h) d s \\
& \geq \alpha_{\xi} h+\alpha_{\lambda} h \int_{\sigma-\ell}^{\sigma} \mu(\sigma, s) d s \\
& \geq h\left(\alpha_{\xi}+\alpha_{\lambda} m_{\ell}\right) \\
& >h=\bar{x}(\sigma),
\end{aligned}
$$

which yields

$$
S \bar{x} \succeq_{\mathcal{C}} \bar{x}
$$

Moreover, for all $\sigma \in \mathbb{R}$ we have

$$
\begin{aligned}
(S \bar{y})(\sigma) & =\xi(\sigma, H)+\int_{\sigma-\ell}^{\sigma} \mu(\sigma, s) \lambda(s, H) d s \\
& \leq \beta_{\xi} H+\beta_{\lambda} H \int_{\sigma-\ell}^{\sigma} \mu(\sigma, s) d s \\
& \leq H\left(\beta_{\xi}+\beta_{\lambda} M_{\ell}\right) \\
& \leq H=\bar{y}(\sigma),
\end{aligned}
$$

which implies that

$$
S \bar{y} \preceq_{C} \bar{y} .
$$

Finally, applying Lemma 1 and observing that any fixed point of $S$ is a solution to (1), the conclusion of Theorem 1 follows.

3.2. Case 2. $\xi(\sigma, \cdot)$ and $\lambda(\sigma, \cdot)$ Are Convex

Theorem 2. Assume that the following conditions hold:

(i) $\quad \mu \in C(\mathbb{R} \times \mathbb{R},[0, \infty))$.

(ii) There exist $0 \leq m_{\ell}<M_{\ell}$, such that

$$
m_{\ell} \leq \int_{\sigma-\ell}^{\sigma} \mu(\sigma, s) d s \leq M_{\ell}, \quad \sigma \in \mathbb{R} .
$$

(iii) There exist $0<h<H$, such that $\xi, \lambda \in C(\mathbb{R} \times[h, H], \mathbb{R})$.

(iv) For all $\sigma \in \mathbb{R}$, the functions $\xi(\sigma, \cdot), \lambda(\sigma, \cdot):[h, H] \rightarrow \mathbb{R}$ are convex and nondecreasing.

(v) There exist $\alpha_{\xi}, \beta_{\xi} \in \mathbb{R}$ and $\alpha_{\lambda}, \beta_{\lambda} \geq 0$, such that

$$
\xi(\sigma, h) \geq \alpha_{\xi} h, \quad \xi(\sigma, H) \leq \beta_{\xi} H, \quad \lambda(\sigma, h) \geq \alpha_{\lambda} h, \quad \lambda(\sigma, H) \leq \beta_{\lambda} H,
$$

for all $\sigma \in \mathbb{R}$.

(vi) $\alpha_{\xi}+\alpha_{\lambda} m_{\ell} \geq 1$ and $\beta_{\xi}+\beta_{\lambda} M_{\ell}<1$.

Then, the integral Equation (1) has a unique continuous solution $\vartheta^{*}$, such that

$$
h \leq \vartheta^{*}(\sigma) \leq H, \quad \sigma \in \mathbb{R} .
$$

Moreover, there exist $\gamma>0$ and $0<\theta<1$ such that, for any continuous function $z_{0}$ satisfying $h \leq z_{0}(\sigma) \leq H, \sigma \in \mathbb{R}$, the sequence $\left\{z_{n}\right\}_{n \geq 0}$ defined by

$$
z_{n+1}(\sigma)=\xi\left(\sigma, z_{n}(\sigma)\right)+\int_{\sigma-\ell}^{\sigma} \mu(\sigma, s) \lambda\left(s, z_{n}(s)\right) d s, \quad \sigma \in \mathbb{R}
$$

converges uniformly to $\vartheta^{*}$ and satisfies

$$
\sup _{\sigma \in \mathbb{R}}\left|z_{n}(\sigma)-\vartheta^{*}(\sigma)\right| \leq \gamma \theta^{n}, \text { for all } n
$$


Proof. From the proof of Theorem 1 , the mapping $S:[\bar{x}, \bar{y}] \rightarrow \mathbb{B}$ is nondecreasing. Using the convexity of $\xi(\sigma, \cdot)$ and $\lambda(\sigma, \cdot)$ (see (iv)), we deduce that $S$ is convex. Moreover, by (10) and (vi), we have

$$
(S \bar{x})(\sigma) \geq h\left(\alpha_{\xi}+\alpha_{\lambda} m_{\ell}\right) \geq h=\bar{x}(\sigma), \quad \sigma \in \mathbb{R}
$$

and

$$
(S \bar{y})(\sigma) \leq H\left(\beta_{\xi}+\beta_{\lambda} M_{\ell}\right)<H=\bar{y}(\sigma), \quad \sigma \in \mathbb{R} .
$$

Then $S \bar{x} \succeq_{\mathcal{C}} \bar{x}$ and $S \bar{y} \preceq_{\mathcal{C}} \bar{y}$. Finally, using Lemma 1, the conclusion of Theorem 2 follows.

\section{Some Examples}

Consider the nonlinear integral equation

$$
\vartheta(\sigma)=2\left(1+e^{-|\sigma|}\right) \sqrt{\vartheta(\sigma)}+\frac{1}{100 \ell} \int_{\sigma-\ell}^{\sigma} e^{-(\sigma-s)^{2}}\left(\frac{3 s^{2} \vartheta(s)}{10\left(s^{2}+1\right)}+(\sin s)^{2} \sqrt{\vartheta(s)}\right) d s, \quad \sigma \in \mathbb{R},
$$

where $\ell>0$ is a constant.

Corollary 1. There exists a unique continuous solution $\vartheta^{*}$ to (12), such that

$$
1 \leq \vartheta^{*}(\sigma) \leq 100, \quad \sigma \in \mathbb{R} .
$$

Moreover, there exist $\gamma>0$ and $0<\theta<1$, such that for any continuous function $z_{0}$ satisfying $1 \leq z_{0}(\sigma) \leq 100, \sigma \in \mathbb{R}$, the sequence $\left\{z_{n}\right\}_{n \geq 0}$ defined by

$$
z_{n+1}(\sigma)=2\left(1+e^{-|\sigma|}\right) \sqrt{z_{n}(\sigma)}+\frac{1}{100 \ell} \int_{\sigma-\ell}^{\sigma} e^{-(\sigma-s)^{2}}\left(\frac{3 s^{2} z_{n}(s)}{10\left(s^{2}+1\right)}+(\sin s)^{2} \sqrt{z_{n}(s)}\right) d s
$$

for all $\sigma \in \mathbb{R}$, converges uniformly to $\vartheta^{*}$ and satisfies

$$
\sup _{\sigma \in \mathbb{R}}\left|z_{n}(\sigma)-\vartheta^{*}(\sigma)\right| \leq \gamma \theta^{n}, \text { for all } n .
$$

Proof. Notice that (12) is a special case of (1) with

$$
\begin{aligned}
& \xi(\sigma, u)=2\left(1+e^{-|\sigma|}\right) \sqrt{u}, \quad \sigma \in \mathbb{R}, u \geq 0 \\
& \mu(\sigma, s)=e^{-(\sigma-s)^{2}}, \quad \sigma, s \in \mathbb{R}, \\
& \lambda(\sigma, u)=\frac{1}{100 \ell}\left(\frac{3 \sigma^{2} u}{10\left(\sigma^{2}+1\right)}+(\sin \sigma)^{2} \sqrt{u}\right), \quad \sigma \in \mathbb{R}, u \geq 0 .
\end{aligned}
$$

Moreover, we have $\mu \in C(\mathbb{R} \times \mathbb{R},[0, \infty))$ and

$$
0 \leq \int_{\sigma-\ell}^{\sigma} \mu(\sigma, s) d s=\int_{\sigma-\ell}^{\sigma} e^{-(\sigma-s)^{2}} d s \leq \int_{\sigma-\ell}^{\sigma} 1 d s=\ell .
$$

This shows that conditions (i) and (ii) of Theorem 1 are satisfied with

$$
m_{\ell}=0 \text { and } M_{\ell}=\ell .
$$

Next, we have $\xi, \lambda \in C(\mathbb{R} \times[0, \infty), \mathbb{R})$, and for all $\sigma \in \mathbb{R}$ the functions

$$
\xi(\sigma, \cdot), \lambda(\sigma, \cdot):[0, \infty) \rightarrow \mathbb{R}
$$

are concave and nondecreasing. Moreover, for all $\sigma \in \mathbb{R}$,

$$
\xi(\sigma, 1)=2\left(1+e^{-|\sigma|}\right) \geq 2, \quad \xi(\sigma, 100)=20\left(1+e^{-|\sigma|}\right) \leq 40<\frac{3}{5} \times 100
$$


and

$$
\lambda(\sigma, 1) \geq 0, \quad \lambda(\sigma, 100)=\frac{1}{100 \ell}\left(\frac{30 \sigma^{2}}{\sigma^{2}+1}+10(\sin \sigma)^{2}\right) \leq \frac{2}{5 \ell}<\frac{1}{5 \ell} \times 100 .
$$

Then, conditions (iv) and (v) of Theorem 1 are satisfied with

$$
h=1, \quad H=100, \quad \alpha_{\xi}=2, \quad \beta_{\xi}=\frac{3}{5}, \quad \alpha_{\lambda}=0, \quad \beta_{\lambda}=\frac{1}{5 \ell} .
$$

Observe also that

$$
\alpha_{\xi}+\alpha_{\lambda} m_{\ell}=\alpha_{\xi}=2>1, \quad \beta_{\xi}+\beta_{\lambda} M_{\ell}=\frac{3}{5}+\frac{1}{5}=\frac{4}{5}<1 .
$$

Then, condition (vi) of Theorem 1 is satisfied. Therefore, the conclusion of Corollary 1 follows from Theorem 1.

Consider now the integral equation

$$
\vartheta(\sigma)=\frac{3}{4 \ell^{3}} e^{-\ell^{2}} \int_{\sigma-\ell}^{\sigma}(\sigma-s)^{2} e^{(\sigma-s)^{2}}\left(\frac{2 s^{2}+1}{s^{2}+1}\right)\left(\vartheta(s)+e^{-\vartheta(s)}\right) d s, \quad \sigma \in \mathbb{R},
$$

where $\ell>0$ is a constant.

Corollary 2. For sufficiently small $h>0$ and sufficiently large $H$, the integral Equation (13) has a unique continuous solution $\vartheta^{*}$ such that

$$
h \leq \vartheta^{*}(\sigma) \leq H, \quad \sigma \in \mathbb{R} .
$$

Moreover, there exist $\gamma>0$ and $0<\theta<1$ such that, for any continuous function $z_{0}$ satisfying $h \leq z_{0}(\sigma) \leq H, \sigma \in \mathbb{R}$, the sequence $\left\{z_{n}\right\}_{n \geq 0}$ defined by

$$
z_{n+1}(\sigma)=\frac{3}{4 \ell^{3}} e^{-\ell^{2}} \int_{\sigma-\ell}^{\sigma}(\sigma-s)^{2} e^{(\sigma-s)^{2}}\left(\frac{2 s^{2}+1}{s^{2}+1}\right)\left(z_{n}(s)+e^{-z_{n}(s)}\right) d s, \quad \sigma \in \mathbb{R}
$$

converges uniformly to $\vartheta^{*}$ and satisfies

$$
\sup _{\sigma \in \mathbb{R}}\left|z_{n}(\sigma)-\vartheta^{*}(\sigma)\right| \leq \gamma \theta^{n}, \text { for all } n .
$$

Proof. Note that (13) is a special case of (1) with

$$
\begin{aligned}
\xi(\sigma, u) & =0, \quad \sigma \in \mathbb{R}, u \geq 0 \\
\mu(\sigma, s) & =(\sigma-s)^{2} e^{(\sigma-s)^{2}}, \quad \sigma, s \in \mathbb{R}, \\
\lambda(\sigma, u) & =\frac{3}{4 \ell^{3}} e^{-\ell^{2}}\left(\frac{2 \sigma^{2}+1}{\sigma^{2}+1}\right)\left(u+e^{-u}\right), \quad \sigma \in \mathbb{R}, u \geq 0 .
\end{aligned}
$$

Moreover, we have $\mu \in C(\mathbb{R} \times \mathbb{R},[0, \infty))$ and

$$
(\sigma-s)^{2} \leq \mu(\sigma, s)=(\sigma-s)^{2} e^{(\sigma-s)^{2}} \leq(\sigma-s)^{2} e^{\ell^{2}}, \quad \sigma \in \mathbb{R}, \sigma-\ell \leq s \leq \sigma,
$$

which yields

$$
\int_{\sigma-\ell}^{\sigma}(\sigma-s)^{2} d s \leq \int_{\sigma-\ell}^{\sigma} \mu(\sigma, s) d s \leq e^{\ell^{2}} \int_{\sigma-\ell}^{\sigma}(\sigma-s)^{2} d s,
$$

that is,

$$
\frac{\ell^{3}}{3} \leq \int_{\sigma-\ell}^{\sigma} \mu(\sigma, s) d s \leq \frac{\ell^{3}}{3} e^{\ell^{2}}
$$


Then, conditions (i) and (ii) of Theorem 2 are satisfied with

$$
m_{\ell}=\frac{\ell^{3}}{3} \quad \text { and } \quad M_{\ell}=\frac{\ell^{3}}{3} e^{\ell^{2}} .
$$

Moreover, we have $\lambda \in C(\mathbb{R} \times[0, \infty), \mathbb{R})$, and for all $\sigma \in \mathbb{R}$ the function

$$
\lambda(\sigma, \cdot):[0, \infty) \rightarrow \mathbb{R}
$$

is convex and nondecreasing. On the other hand, taking a sufficiently small $h>0$ so that

$$
1+\frac{e^{-h}}{h} \geq 4 e^{\ell^{2}}
$$

it holds that

$$
\lambda(\sigma, h)=\frac{3}{4 \ell^{3}} h\left[e^{-\ell^{2}}\left(\frac{2 \sigma^{2}+1}{\sigma^{2}+1}\right)\left(1+\frac{e^{-h}}{h}\right)\right] \geq \frac{3}{\ell^{3}} h, \quad \sigma \in \mathbb{R} .
$$

Next, taking $H$ that is sufficiently large so that

$$
\frac{e^{-H}}{H}<\frac{1}{2}
$$

we obtain

$$
\lambda(\sigma, H)=\frac{3}{4 \ell^{3}} e^{-\ell^{2}}\left(\frac{2 \sigma^{2}+1}{\sigma^{2}+1}\right)\left(H+e^{-H}\right) \leq \frac{3}{2 \ell^{3}} e^{-\ell^{2}} H\left(1+\frac{e^{-H}}{H}\right) \leq \frac{9}{4 \ell^{3}} e^{-\ell^{2}} H .
$$

Therefore, the conditions (iv) and (v) of Theorem 2 are satisfied with

$$
\alpha_{\xi}=\beta_{\xi}=0, \quad \alpha_{\lambda}=\frac{3}{\ell^{3}}, \quad \beta_{\lambda}=\frac{9}{4 \ell^{3}} e^{-\ell^{2}} .
$$

We have also

$$
\alpha_{\xi}+\alpha_{\lambda} m_{\ell}=\frac{3}{\ell^{3}} \frac{\ell^{3}}{3}=1, \quad \beta_{\xi}+\beta_{\lambda} M_{\ell}=\frac{9}{4 \ell^{3}} e^{-\ell^{2}} \frac{\ell^{3}}{3} e^{\ell^{2}}=\frac{3}{4}<1 .
$$

Then, condition (vi) of Theorem 2 is satisfied. Finally, the conclusion of Corollary 2 follows from Theorem 2.

\section{Conclusions}

The integral Equation (1) is investigated in this paper. Using some techniques from partial-ordering methods and a fixed-point theorem for concave (and convex) monotone operators (see Lemma 1), the existence and uniqueness of positive solutions is proved. Namely, we investigated two cases. In the first case, it is supposed that $\xi(\sigma, \cdot)$ and $\lambda(\sigma, \cdot)$ are concave functions. In the second case, $\xi(\sigma, \cdot)$ and $\lambda(\sigma, \cdot)$ are supposed to be convex. In both cases, sufficient conditions ensuring the existence and uniqueness of positive solutions are provided, as well as a numerical algorithm converging to the solution (see Theorems 1 and 2). We also provided some examples to illustrate our results (see Section 4). Comparing these with the existence results from the literature, to the best of our knowledge, the study of (1) with convex and concave nonlinearities was not previously investigated.

Author Contributions: R.P.A., M.J. and B.S. contributed equally to this paper. All authors have read and agreed to the published version of the manuscript.

Funding: The second author is supported by Researchers Supporting Project number (RSP-2021/57), King Saud University, Riyadh, Saudi Arabia. 
Conflicts of Interest: The authors declare no conflict of interest.

\section{References}

1. Cooke, K.L.; Kaplan, J.L. A periodicity threshold theorem for epidemics and population growth. Math. Biosci. 1976, 31, 87-104. [CrossRef]

2. Leggett, R.W.; Williams, L.R. A fixed point theorem with application to an infectious disease model. J. Math. Anal. Appl. 1980, 76, 91-97. [CrossRef]

3. Williams, L.R.; Leggett, R.W. Nonzero solutions of nonlinear integral equations modeling infectious disease. SIAM J. Math. Anal. 1982, 13, 112-121. [CrossRef]

4. Guo, D.; Lakshmikantham, K. Positive solutions of nonlinear integral equations arising in infectious disease. J. Math. Anal. Appl. 1988, 134, 1-8. [CrossRef]

5. Nussbaum, R.D. A periodicity threshold theorem for some nonlinear integral equations. SIAM J. Math. Anal. 1978, 9, 356-376. [CrossRef]

6. Smith, H.L. An abstract threshold theorem for one parameter families of positive noncompact operators. Funkcial. Ekuuc. 1981, 24, 141-153.

7. Chen, S.; Torrejón, R. Bifurcation of almost periodic solutions for a nonlinear integral equation with delay. Nonlinear Anal. 1996, 27, 863-877. [CrossRef]

8. Dads, E.A.; Ezzinbi, K. Existence of positive pseudo almost periodic solution for a class of functional equations arising in epidemic problems. Cybernet. Syst. Anal. 1994, 30, 900-910. [CrossRef]

9. Ezzinbi, K.; Hachimi, M.A. Existence of positive almost periodic solutions of functional equations via Hilbert's projective metric. Nonlinear Anal. 1996, 26, 1169-1176. [CrossRef]

10. Fink, A.M.; Gatica, J.A. Positive almost periodic solutions of some delay integral equations. J. Differ. Equ. 1990, 83, 166-178. [CrossRef]

11. Torrejón, R. Positive almost periodic solutions of a state-dependent delay nonlinear integral equation. Nonlinear Anal. 1993, 20, 1383-1416. [CrossRef]

12. Dads, E.A.; Ezzinbi, K. Almost periodic solution for some neutral nonlinear integral equation. Nonlinear Anal. 1997, 28, 1479-1489. [CrossRef]

13. Agarwal, R.P.; O'Regan, D. Periodic solutions to nonlinear integral equations on the infinite interval modelling infectious disease. Nonlinear Anal. 2000, 40, 21-35. [CrossRef]

14. Agarwal, R.P.; O’Regan, D.; Wong, P.J.Y. Dynamics of epidemics in homogeneous/heterogeneous populations and the spreading of multiple inter-related infectious diseases: constant-sign periodic solutions for the discrete model. Nonlinear Anal. 2007, 8 , 1040-1061. [CrossRef]

15. Dads, E.A.; Ezzinbi, K. Existence of positive pseudo-almost-periodic solution for some nonlinear infinite delay integral equations arising in epidemic problems. Nonlinear Anal. 2000, 41, 1-13. [CrossRef]

16. Ding, H.S.; Chen, Y.Y.; N'Guérékata, G.M. Existence of positive pseudo almost periodic solutions to a class of neutral integral equations. Nonlinear Anal. 2011, 74, 7356-7364. [CrossRef]

17. Dobriţoiu, M.; Şerban, M.-A. Step method for a system of integral equations from biomathematics. Appl. Math. Comput. 2014, 227, 412-421. [CrossRef]

18. Jleli, M.; Samet, B. Global existence of solutions to a system of integral equations related to an epidemic model. J. Funct. Spaces 2020, 6625525. [CrossRef]

19. Kang, S. Existence and uniqueness of positive periodic solutions for a class of integral equations with mixed monotone nonlinear terms. Appl. Math. Lett. 2017, 71, 24-29. [CrossRef]

20. Li, M.Y. An Introduction to Mathematical Modeling of Infectious Diseases; Springer: Berlin, Germany, 2018.

21. Wang, C.H.; Ding, H.S.; N'Guérékata, G.M. Existence of positive solutions for some nonlinear quadratic integral equations. Electron. J. Differ. Equ. 2019, 79, 1-11.

22. Zhao, J.Y.; Ding, H.S.; N'Guérékata, G.M. Positive almost periodic solutions to integral equations with superlinear perturbations via a new fixed point theorem in cones. Electron. J. Differ. Equ. 2017, 2, 1-10.

23. Sidorov, D.N. Existence and blow-up of Kantorovich principal continuous solutions of nonlinear integral equations. Differ. Equat. 2014, 50, 1217-1224. [CrossRef]

24. Sidorov, D.N. Integral Dynamical Models: Singularities, Signals \& Control; World Scientific Series on Nonlinear Science Series A; Chua, L.O., Ed.; World Scientific Publ. Pte Ltd.: Singapore, 2015; Volume 87.

25. Panin, A.A. On local solvability and blow-up of solutions of an abstract nonlinear Volterra integral equation. Math. Notes 2015, 97, 892-908. [CrossRef]

26. Noeiaghdam, S.; Sidorov, D.; Wazwaz, A.-M.; Sidorov, N.; Sizikov, V. The Numerical Validation of the Adomian Decomposition Method for Solving Volterra Integral Equation with Discontinuous Kernels Using the CESTAC Method. Mathematics. 2021, 9, 260. [CrossRef]

27. Guo, D.; Cho, Y.J.; Zhu, J. Partial Ordering Methods in Nonlinear Problems; Nova Science: New York, NY, USA, 2004. 